\title{
Near-IR Spectroscopy of High-z QSOs: Relating the Broad and Narrow-Line Regions
}

\author{
M.S. Brotherton \\ The University of Texas, Austin, TX 78712, USA
}

\section{Introduction}

Recent investigations of the broad UV lines in luminous QSOs identified strong correlations involving emission-line widths, shifts, equivalent widths, and ratios (Francis et al. 1992; Wills et al. 1993; Brotherton et al. 1994a, b). A simple model developed to explain these trends approximates UV broad lines as emission from two regions, an intermediate-line region (ILR), and a very broad-line region (VBLR), together comprising the traditional broad-line region (BLR). The observed and derived properties for the ILR and VBLR are summarized in Table 1, along with typical values for the narrow-line region (NLR).

Table 1. Comparison of Emission-Line Regions

\begin{tabular}{lccc}
\hline \hline & NLR & ILR & VBLR \\
\hline Velocity Dispersion $\left(\mathrm{km} \mathrm{s}^{-1}\right)$ & $\sim 500$ & $\sim 2000$ & $\sim 7000$ \\
Radial Distance $(\mathrm{pc})$ & $10^{2-3}$ & $\sim 1$ & $\sim 0.1$ \\
Gas Density $\left(n_{\mathrm{H} \mathrm{cm}} \mathrm{cm}^{-3}\right)$ & $10^{4-6}$ & $\sim 10^{10}$ & $\sim 10^{12.5}$ \\
Ionization Parameter $\left(U=\phi_{i} / n_{\mathrm{H}}\right)$ & $\sim 0.01$ & $\sim 0.01$ & $\sim 0.01$ \\
Redshift cf. Systemic $\left(\mathrm{km} \mathrm{s}^{-1}\right)$ & 0 & $\sim 0$ & $\sim-1000$ \\
Covering Factor $(\Omega / 4 \pi)$ & $\leq 0.02$ & $\leq 0.03$ & $\sim 0.24$ \\
\hline
\end{tabular}

To test the hypothesis that the ILR is related to the NLR, I obtained spectra of the $\mathrm{H} \beta-\left[\mathrm{O}_{\mathrm{III}}\right] \lambda 5007$ region for 32 QSOs studied by Brotherton et al. (1994a) and Steidel \& Sargent (1991) (see Fig. 1, left). Narrow Cirl] $\lambda 1909$ indicates significant ILR emission, while large $\left[\mathrm{O}_{\mathrm{III}}\right] \lambda 5007 / \mathrm{H} \beta$ indicates significant NLR emission, so based on the above hypothesis I predicted an inverse correlation between FWHM of $\mathrm{C}$ III] $\lambda 1909$ and [O III] $\lambda 5007 / \mathrm{H} \beta$ (Brotherton et al. 1994b).

\section{Results and Discussion}

The predicted trend is present (Fig. 1, top right), indicating that ILR and NLR emission are correlated. Furthermore, the strength of optical Fe Il emission is inversely correlated with the strength of ILR and NLR emission (Fig. 1, bottom right). These $\sim 4 \sigma$ correlations are similar if the $[\mathrm{O}$ III] and Fe II line strengths are normalized by the continuum rather than $H \beta$.

These results show that the first eigenvectors of the principal component analyses of Francis et al. (1992) and Boroson \& Green (1992) are the same, hence 

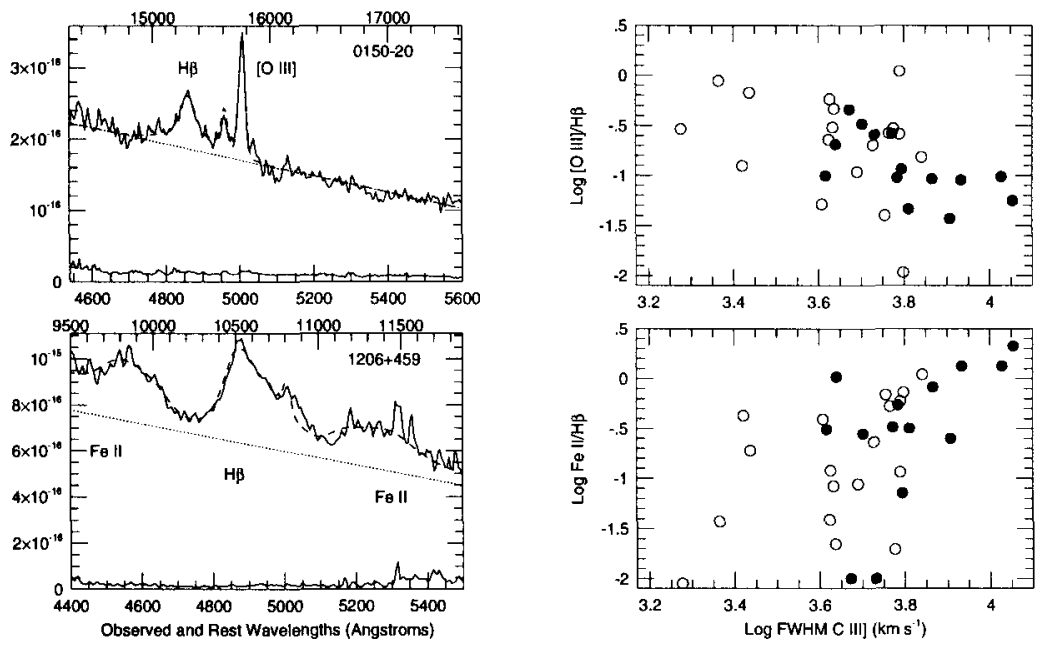

Figure 1. Left: Example flux and error spectra, with fits used to make measurements. Right: Radio-loud, open; radio-quiet, filled.

relating many QSO properties, including radio and X-ray emission. Variation along this sequence may constitute a 'fundamental plane' for QSOs. Explanations for the underlying cause of these related trends have included the variation of viewing angle, continuum shape, BLR size, radio-jet power, and the physical properties of BLR clouds. I favor the last item, specifically the percentage of optically thick VBLR clouds. A modest covering factor can largely cover typical biconical geometries. Optically thick VBLR clouds can simultaneously produce Fe II emission and shadow more distant clouds, extinguishing the continuum seen by the ILR and NLR.

Acknowledgments. I thank Bev Wills, Derek Wills, the staffs of McDonald Observatory, KPNO, and CTIO, Jack Baldwin, Gary Hill, Chuck Steidel, Wal Sargent, Keith Thompson, Richard Elston, Jerry Kriss, and the AAS.

\section{References}

Boroson, T. A., \& Green, R.F. 1992, ApJS, 80, 109.

Brotherton, M.S., Wills, B. J., Steidel, C.C., \& Sargent, W. L. W. 1994a, ApJ, $423,131$.

Brotherton, M.S., Wills, B.J., Francis, P. J., \& Steidel, C. C. 1994b, ApJ, 430, 495.

Francis, P. J., Hewett, P. C., Foltz, C. B., \& Chaffee, F. H. 1992, ApJ, 398, 476.

Steidel, C. C., \& Sargent, W.L.W. 1991, ApJ, 382, 433.

Wills, B.J., Brotherton, M.S., Fang, D., Steidel, C.C., \& Sargent, W.L.W. 1993, ApJ, 415, 563. 